\title{
The B-type variable HD 131120 modelled by rotational modulation ${ }^{\star}$
}

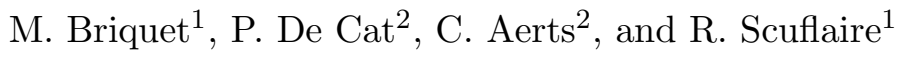 \\ 1 Institut d'Astrophysique et de Géophysique de Liège, Université de Liège, avenue de Cointe 5, 4000 Liège, \\ Belgium \\ 2 Instituut voor Sterrenkunde, Katholieke Universiteit Leuven, Celestijnenlaan 200 B, 3001 Heverlee, Belgium
}

Received 6 June 2001 / Accepted 17 September 2001

\begin{abstract}
We present ground-based multi-colour Geneva photometry and high-resolution spectra of the variable B-type star HD 131120. All sets of data reveal a monoperiodic star with a period of 1.569 days. We compare the variability of the star with a non-radial $g$-mode pulsation model and with a rotational modulation model. The latter model appears to be the best explanation for the observed line-profile variations of the star. Moreover, contrary to the pulsational model, it is able to reproduce the behaviour of the first three moments of the $\mathrm{Si}$ and He lines as well as the large variability of their equivalent width. We therefore remove the star from the list of new Slowly Pulsating B stars found from Hipparcos data.
\end{abstract}

Key words. stars: early-type - stars: variables - stars: individual: HD 131120

\section{Introduction}

From the Hipparcos mission a huge number of B-type stars have been classified as new Slowly Pulsating B stars (SPBs) by Waelkens et al. (1998). A sample among these SPBs has been selected for a long-term spectroscopic and photometric monitoring (see Aerts et al. 1999; De Cat et al. 2000). This paper is devoted to the analysis of one of these stars: HD 131120.

Aerts et al. (1999) denoted that the same main frequency of $0.6374 \mathrm{c} / \mathrm{d}$ was found in the Hipparcos and Geneva photometry and in the first moment (which is equivalent to the radial velocity) of the Si II 4128-4130 $\AA$ lines (see Aerts et al. 1992 for a definition of the moments of a line profile). In Aerts et al. (1999) it is pointed out that only one frequency is found in the data of this star, while other SPBs are multiperiodic. Moreover, the first moment is non-sinusoidal: the first harmonic of the frequency found is necessary to obtain a good fit. In fact, this star is not the only one to present these peculiarities. In Briquet et al. (2001) we studied the B-type star HD 105382. We showed that this star is situated in the upper and blue part of the SPB instability strip, that all sets of photometric and spectroscopic data reveal a monoperiodic star, and that the first moment of the Si II

Send offprint requests to: M. Briquet, e-mail: briquet@astro.ulg.ac.be

* Based on observations obtained with the Swiss photometric telescope and ESO's CAT/CES telescope, both situated at La Silla, Chile.
4128-4130 A lines is non-sinusoidal. We tried to interpret the variability of this star in terms of a non-radial $g$-mode pulsation model but also in terms of a rotational modulation model, since the observed period is compatible with the rotation period of a star with such a spectral type. Unfortunately, neither of these two models was able to explain the observed line-profile variations.

In this paper we compare the observed photometric data and line-profile variations of HD 131120 with a nonradial pulsation (NRP) model and with a spot model. The paper is organized as follows. In Sect. 2 the description of the data and some physical parameters are given. In Sect. 3 the result of the frequency analysis on the data is described. In Sect. 4 we attempt to perform a mode identification. We do this for the photometric data using the method of photometric amplitudes. For the spectroscopic data we use both the moment method and line-profile fitting. Then we try to model the variations with a rotational modulation model (Sect. 5). The outcome of the modelling of the variations of the star is discussed in Sect. 6 .

\section{Data}

We have three data sets at our disposal: Hipparcos photometry, multicolour Geneva photometry and highresolution spectroscopic data. The Geneva photometric observations were obtained with the Swiss telescope situated at La Silla in Chile, during several 3-weeks runs in the course of 1997. The spectroscopic data were obtained with the CAT/CES telescope of ESO in Chile, during 
Table 1. Observing log for Geneva photometry (upper) and spectroscopy (lower).

\begin{tabular}{ccc}
\hline $\begin{array}{c}\text { Number of } \\
\text { observations }\end{array}$ & \multicolumn{2}{c}{ JD } \\
& Start & End \\
\hline 32 & 485 & 503 \\
69 & 541 & 583 \\
7 & 588 & 601 \\
\hline \hline 27 & 163 & 170 \\
15 & 188 & 194 \\
13 & 272 & 277 \\
4 & 490 & 498 \\
4 & 513 & 528 \\
6 & 571 & 576 \\
8 & 633 & 638 \\
\hline
\end{tabular}

7 separate weeks of monitoring spread over 1996-1998. The spectral domain was $[4115,4135] \AA$ in order to get the SiII-doublet with lines at $\lambda \lambda 4128,4130 \AA$. The signalto-noise ratio is about 400 . The number of observations and the ranges of their Julian Dates are given in Table 1 for the Geneva photometry and the spectroscopy. For a complete description of the observations and data reductions we refer to Aerts et al. (1999) and De Cat (2001). We point out that the data published in this work is available upon request from the authors.

In Aerts et al. (1999) some physical parameters of the star are given. They are:

$$
\left\{\begin{array}{l}
\log T_{\text {eff }}=4.26 \pm 0.01 \\
\log g=4.1 \pm 0.15 \\
\log L / L_{\odot}=3.13 \pm 0.15 \\
M=6.1 M_{\odot}, \\
R=3.6 R_{\odot} .
\end{array}\right.
$$

De Geus et al. (1989) found similar values for the parameters of the star: $\log T_{\text {eff }}=4.27, \log g=4.22$ and $\log L / L_{\odot}=3$. In Borra et al. (1983), a photometric spectral type with observed $U B V$ colors is given for the star, which is B 2.5. With these parameters, HD 131120 is situated in the upper part of the SPB instability domain, close to the $\beta$ Cep instabilitsy domain (see e.g. Pamyatnykh 1999).

\section{Frequency analysis}

The frequency analysis on the three data sets was performed using the PDM method (Stellingwerf 1978) and the CLEAN method (Roberts et al. 1987). We tested frequencies from 0 to 3 cycles per day (c/d) with a frequency step of $0.0001 \mathrm{c} / \mathrm{d}$ and we searched for multiple periods by prewhitening.

The frequency of $0.6374 \mathrm{c} / \mathrm{d}$ is found in the Hipparcos photometry. The same frequency is obtained from the Geneva data in the 7 filters. In Table 2 the standard deviation of the data in the different filters is given as well as

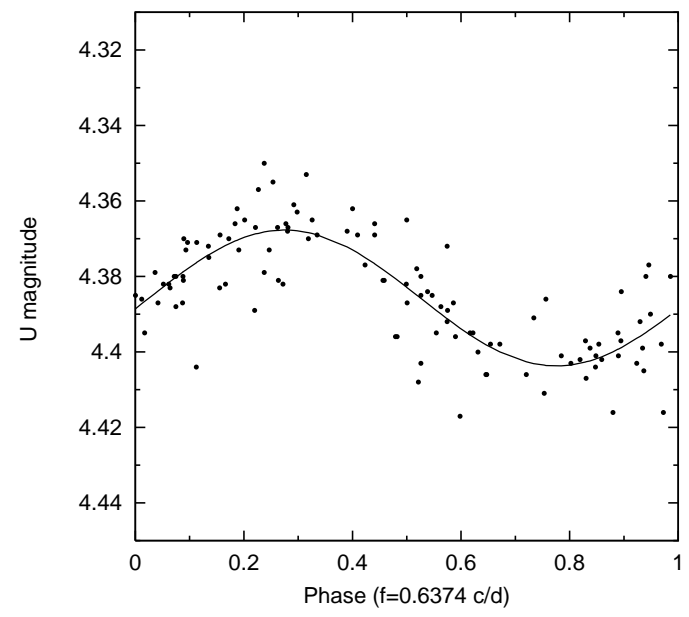

Fig. 1. Phase diagram of the $U$-magnitude data.

Table 2. Standard deviation of the Geneva data in the different filters and of the residuals after fitting with a sine for the frequency $0.6374 \mathrm{c} / \mathrm{d}$.

\begin{tabular}{ccc}
\hline Filter & \multicolumn{2}{c}{ Standard deviation } \\
& Data & Residuals \\
\hline$U$ & 0.0151 & 0.0091 \\
$B_{1}$ & 0.0120 & 0.0072 \\
$B$ & 0.0106 & 0.0065 \\
$B_{2}$ & 0.0111 & 0.0073 \\
$V_{1}$ & 0.0090 & 0.0058 \\
$V$ & 0.0091 & 0.0055 \\
$G$ & 0.0096 & 0.0059 \\
\hline
\end{tabular}

the one of the residuals after fitting with a sine. A phase diagram for the $U$-filter is shown in Fig. 1. This frequency reduces the standard deviation in the $U$ filter by $40 \%$. An additional frequency cannot be found either in the Hipparcos data or in the Geneva data.

From the doublet Si II centered at $\lambda \lambda 4128,4130 \AA$ we computed the first moment and performed a frequency analysis. We again obtained the frequency $0.6374 \mathrm{c} / \mathrm{d}$. A sine fit to the first moment for this frequency explains $67 \%$ of the standard deviation and a slightly better fit leading to a reduction in standard deviation of $69 \%$ is obtained with its first harmonic included (see upper panel of Fig. 2). No other frequency can be found in the data and it is clear that this star is monoperiodic.

We note that, if the variability is due to pulsation, such a long period is attributed to a $g$-mode. We also note that the observed period may be the rotation period of the star.

\section{Non-radial pulsation model}

\subsection{The Geneva photometric data}

The method of photometric amplitudes as described by Watson (1988) allows us to derive the degree $\ell$ of the pulsation mode from multicolour photometry by comparing the 
Table 3. Amplitudes of the least-squares sine fits to the Geneva data in the different filters, computed together with their standard errors using the SAS-software package. Ratios of the amplitudes with respect to the $U$-filter are also listed.

\begin{tabular}{ccc}
\hline Filter & Amplitude & Ratio \\
\hline$U$ & $0.0183 \pm 0.0013$ & 1 \\
$B_{1}$ & $0.0145 \pm 0.0010$ & $0.792 \pm 0.077$ \\
$B$ & $0.0125 \pm 0.0009$ & $0.685 \pm 0.069$ \\
$B_{2}$ & $0.0123 \pm 0.0010$ & $0.672 \pm 0.072$ \\
$V_{1}$ & $0.0103 \pm 0.0008$ & $0.566 \pm 0.060$ \\
$V$ & $0.0109 \pm 0.0008$ & $0.599 \pm 0.058$ \\
$G$ & $0.0111 \pm 0.0008$ & $0.608 \pm 0.061$ \\
\hline
\end{tabular}

observed amplitudes at some wavelengths (i.e. those of the central wavelengths of the passbands of the used photometric system) with the theoretical amplitudes calculated for several values of $\ell$ and a free parameter $S$ taking into account non-adiabatics effects $(S \in[0,1], 0$ : fully nonadiabatic, 1: adiabatic). We note that the parameter $S$ is the same as the parameter $R$ of Stamford \& Watson (1981). As the theoretical amplitudes are proportional to an unknown wavelength-independent function, ratios of the amplitudes are considered in order to eliminate this function. In Table 3 we give the amplitudes obtained with a sine fit for the separate Geneva filters, together with the amplitude ratios with respect to the $U$-filter. We refer to Heynderickx et al. (1994) for a full description of the method we used in this work and to Briquet et al. (2001) for a brief description. In general the mode identification by this method is successful for $\beta$ Cep stars (see e.g. Heynderickx et al. 1994) and SPBs (De Cat 2001). For HD131120 we tested $\ell$ from 0 to 7 and we choose the degree $\ell$ using a discriminant $\eta_{\ell}(S)$. This discriminant is the square root of the sum of squares of the differences between the observed and theoretical amplitude ratios divided by 7 . For each $\ell$ we determine the value of $S$ for which the discriminant $\eta_{\ell}(S)$ is minimal. Then we choose the mode for which the discriminant attains the lowest value. The minima of the discriminant $\eta_{\ell}$ are given in the left columns of Table 4 . The best solutions are $\ell=1,2,4$ and 6 . They all have very similar values for the discriminant and so are equivalent in quality. Moreover the derived amplitude ratios have a large uncertainty (see Table 3 ). This observational uncertainty is larger than the difference between the competing pulsational models. We then conclude that the photometric data do not allow us to determine the degree $\ell$.

\subsection{The spectroscopic data}

\subsubsection{Mode identification by the moment method}

The moment method is the most objective criterion to identify modes of non-radial pulsators with a slow rotation. The identification of modes is performed by
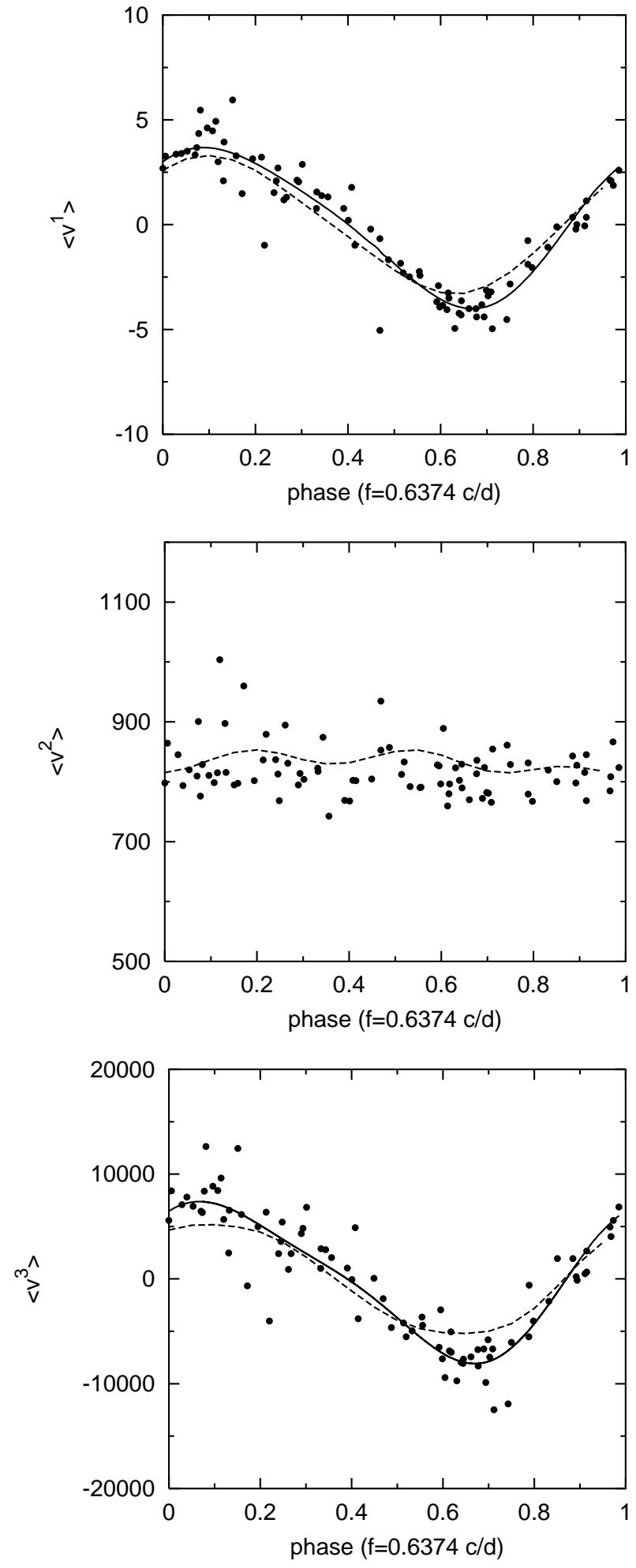

Fig. 2. Phase diagram of the first three moments of the Si II $4128 \AA$ line. We show the observed values (dots), the fit using $0.6374 \mathrm{c} / \mathrm{d}$ and its first harmonic (solid line), and the best spot model (dashed line).

comparing the amplitudes of the first three observed moments of a line profile $\left\langle v^{1}\right\rangle,\left\langle v^{2}\right\rangle$ and $\left\langle v^{3}\right\rangle$ (see Aerts et al. 1992) with theoretically calculated ones by means of a 


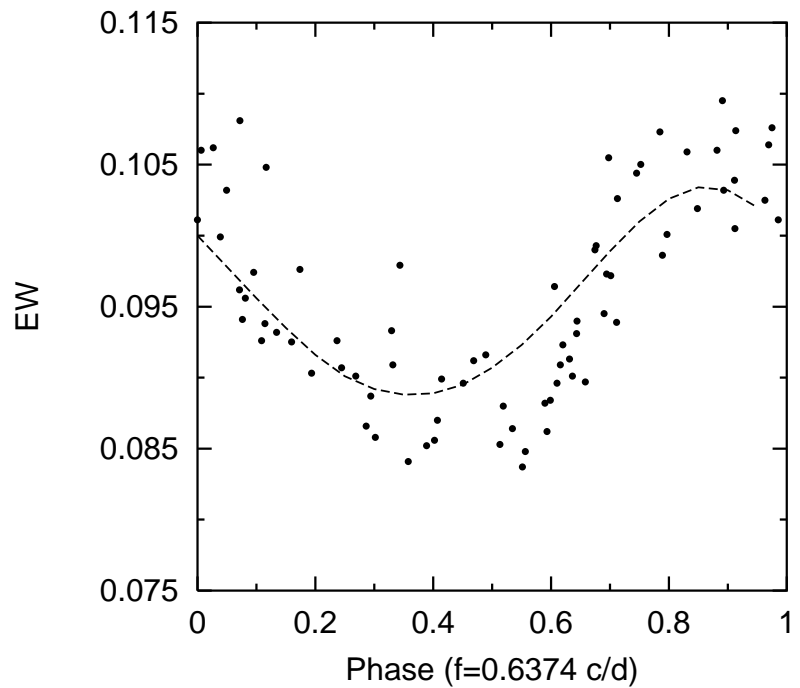

Fig. 3. Phase diagram of the equivalent width of the SiII $4128 \AA$ line, which is expressed in $\AA$. The comparison with the best spot model is represented in dashed line.

discriminant. For a full description of the method we refer to Aerts (1996).

We calculated the first three observed moments of a line profile $\left\langle v^{1}\right\rangle,\left\langle v^{2}\right\rangle$ and $\left\langle v^{3}\right\rangle$ (see Aerts et al. 1992). The first moment is equivalent to the radial velocity, for which we only found the frequency $f=0.6374 \mathrm{c} / \mathrm{d}$. The linear pulsation theory predicts that $\left\langle v^{2}\right\rangle$ varies with both $f$ and $2 f$ while $\left\langle v^{3}\right\rangle$ varies with $f, 2 f$ and $3 f$. In order to compare the frequencies found in the observed moments to the theoretical predictions we performed a frequency analysis on the observed moments. In the second moment, it was not possible to determine a frequency and in the third moment we found only the frequency $f=0.6374 \mathrm{c} / \mathrm{d}$. A phase diagram for the frequency $f=0.6374 \mathrm{c} / \mathrm{d}$ for $\left\langle v^{1}\right\rangle$, $\left\langle v^{2}\right\rangle$ and $\left\langle v^{3}\right\rangle$ is shown in Fig. 2. It is clear that the second moment does not vary with $f$ nor with $2 f$. Such a situation does not correspond to linear pulsation theory as described above. We also computed the moment of order zero, which is the equivalent width of the line. The same frequency of $0.6374 \mathrm{c} / \mathrm{d}$ is present in it and a phase diagram is shown in Fig. 3. We point out that the relative $E W$ variation is about $10 \%$. Such a large value is not encountered for confirmed SPBs (see De Cat 2001).

The discriminant is a function of the differences between the observed and theoretically calculated amplitudes of the first three moments. For each set of wavenumbers $(\ell, m)$ we determine the values of $v_{\mathrm{p}}, i, v_{\Omega}$ and $\sigma$ for which the discriminant $\Gamma_{\ell}^{m}\left(v_{\mathrm{p}}, i, v_{\Omega}, \sigma\right)$ is minimal. Then we chose the mode $(\ell, m)$ for which the discriminant attains the lowest value. The outcome of the mode identification with the discriminant is listed in the right columns of Table 4 for the best solutions in parameter space. We tested $\ell$ from 0 to 6 because the discriminant is only able to correctly identify modes with low to moderate degree. The other velocity parameters were varied in the interval $[0.1 ; 2] \mathrm{km} \mathrm{s}^{-1}$ with a step $0.1 \mathrm{~km} \mathrm{~s}^{-1}$ for the amplitude of
Table 4. Left: the different minima of the discriminant $\eta_{\ell}$ of the method of photometric amplitudes together with the most likely value of the free parameter $S$ taking into account nonadiabatic effects. Right: the different minima of the discriminant $\Gamma_{\ell}^{m}$ of the moment method for the Si II $4128 \AA$ line for the best solutions. $v_{\mathrm{p}}$ is the amplitude of the radial part of the pulsation velocity, expressed in $\mathrm{km} \mathrm{s}^{-1} ; i$ is the inclination angle; $v_{\Omega}$ is the projected rotational velocity, expressed in $\mathrm{km} \mathrm{s}^{-1}$ and $\sigma$ is the intrinsic line-profile width, also expressed in $\mathrm{km} \mathrm{s}^{-1}$.

\begin{tabular}{ccc|ccccccc}
\hline$\ell$ & $\eta_{\ell}$ & $S$ & $\ell$ & $m$ & $\Gamma_{\ell}^{m}$ & $v_{\mathrm{p}}$ & $i$ & $v_{\Omega}$ & $\sigma$ \\
\hline 1 & 0.025 & 0.09 & 3 & 0 & 0.30 & 0.2 & $63^{\circ}$ & 57 & 5 \\
4 & 0.034 & 0.14 & 3 & \pm 1 & 0.31 & 0.2 & $33^{\circ}$ & 57 & 3 \\
2 & 0.038 & 1.00 & 1 & 0 & 0.34 & 0.5 & $76^{\circ}$ & 41 & 20 \\
6 & 0.068 & 0.07 & 1 & \pm 1 & 0.38 & 0.8 & $11^{\circ}$ & 57 & 2 \\
5 & 0.133 & 1.00 & 3 & \pm 2 & 0.39 & 0.3 & $73^{\circ}$ & 44 & 17 \\
7 & 0.135 & 1.00 & 2 & 0 & 0.43 & 0.4 & $68^{\circ}$ & 48 & 15 \\
3 & 0.172 & 1.00 & 2 & \pm 2 & 0.46 & 0.2 & $31^{\circ}$ & 53 & 12 \\
& & & $\vdots$ & $\vdots$ & $\vdots$ & $\vdots$ & $\vdots$ & $\vdots$ & $\vdots$ \\
\hline
\end{tabular}

the radial part of the pulsation velocity $v_{\mathrm{p}},\left[1^{\circ} ; 90^{\circ}\right]$ with a step $1^{\circ}$ for the inclination angle $i,[40 ; 70] \mathrm{km} \mathrm{s}^{-1}$ with a step $1 \mathrm{~km} \mathrm{~s}^{-1}$ for the projected rotational velocity $v_{\Omega}$ and $[1 ; 20] \mathrm{km} \mathrm{s}^{-1}$ with a step $1 \mathrm{~km} \mathrm{~s}^{-1}$ for the intrinsic lineprofile width $\sigma$. The more probable mode is $(\ell, m)=(3,0)$. However, there are other candidates of almost equal probability, as can be seen in Table 4 . We point out that the moment method is not able to distinguish the sign of the azimuthal number $m$.

Unfortunately no confidence intervals for the minima of the discriminant and the corresponding velocity parameters $v_{\mathrm{p}}, i, v_{\Omega}$ and $\sigma$ can be determined. Consequently, we generated theoretical line-profile variations for the modes for which the discriminant attains the lowest value (see Table 4) in order to choose the mode which gives the best fit compared to the observed line-profile variations. We define the "best fit model" as the one which has the smallest standard deviation in the intensity over all profiles $\Sigma \equiv \frac{1}{N} \sum_{j=1}^{N} \sqrt{\frac{1}{n_{j}} \sum_{i=1}^{n_{j}}\left(I_{i, \text { obs }}^{j}-I_{i, \text { th }}^{j}\right)^{2}}$, with $N$ the number of spectra and $n_{j}$ the number of wavelength pixels in the spectra $j$. We found that $\Sigma$ has about the same value for the most likely modes listed in Table 4, which is 0.004 and we are then again not able to determine the most likely mode from the discriminant.

\subsubsection{Mode identification by line-profile fitting}

Another way to identify modes from line profiles is to compare the observed line-profile variations with theoretically calculated ones. At present the best code which simulates line profiles for a rotating star undergoing NRP is Townsend's (1997) code, called BRUCE, which includes the Coriolis force. Unlike the previous ones, this code is valid for all the ratios $\Omega / \omega$ of the rotation and pulsation frequencies and not only for $\Omega / \omega$ smaller than unity. 
Up to now, in BRUCE, temperature variations are taken to be adiabatic and two extra parameters must be included to simulate non-adiabatic temperature effects.

We search for the parameters for which the calculated profiles best fit the observed profiles by considering a large grid of possible wavenumbers and parameters. In order to keep the computation time feasible we averaged out all the observed profiles in phase bins of 0.05 of the variability cycle and worked with these 20 averaged observed profiles. They are shown as dotted lines in Fig. 4. The observed profiles are compared to the theoretical profiles and to their orthogonal symmetric profiles in order not to favour a sense of rotation. As a measure of the goodness of fit we use the standard deviation $\Sigma$ in the intensity averaged over all profiles. The most likely mode and parameters are those that minimize $\Sigma$.

First we consider only the velocity perturbation and we cover the parameter space by varying the free parameters in the following way: $\ell$ from 0 to 6 , the projected rotational velocity $v_{\Omega}$ from 30 to $60 \mathrm{~km} \mathrm{~s}^{-1}$ with a step $5 \mathrm{~km} \mathrm{~s}^{-1}$, the angle of inclination $i$ between the rotation axis and the line of sight $i$ from $10^{\circ}$ to $90^{\circ}$ with a step $10^{\circ}$, the amplitude of the radial part of the pulsation velocity $v_{\mathrm{p}}$ from 2 to $20 \mathrm{~km} \mathrm{~s}^{-1}$ with a step $2 \mathrm{~km} \mathrm{~s}^{-1}\left(v_{\mathrm{p}}\right.$ from 0.5 to $1.5 \mathrm{~km} \mathrm{~s}^{-1}$ with a step $0.5 \mathrm{~km} \mathrm{~s}^{-1}$ for modes with $m=0$ ), the intrinsic line-profile width $\sigma$ from 2.5 to $20 \mathrm{~km} \mathrm{~s}^{-1}$ with a step $2.5 \mathrm{~km} \mathrm{~s}^{-1}$, the initial phase of the mode $\phi$ from 0 to 0.95 period with a step 0.05 period. Then we consider adiabatic temperature variations and used theoretical intrinsic profiles kindly provided by Dr. T. Rivinius. These are constructed using the atmospheric codes ATLAS 9 and BHT (Baschek-Holweger-Traving, see Gummersbach et al. 1998) and fixing the microturbulence at $2 \mathrm{~km} \mathrm{~s}^{-1}$. Finally we consider non-adiabatic temperature effects by introducing two extra parameters which are the non-adiabatic temperature perturbation scaling factor $\Delta T$ and the non-adiabatic temperature perturbation phase shift $\psi$. We take $\Delta T$ from 0.2 to 1 with a step 0.2 and $\psi$ from $0^{\circ}$ to $360^{\circ}$ with a step $45^{\circ}$.

The parameters that give the smallest $\Sigma$ are:

$$
\left\{\begin{array}{l}
\ell=2 \\
m=0 \\
v_{\Omega}=55 \mathrm{~km} \mathrm{~s}^{-1}, \\
i=90^{\circ} \\
v_{\mathrm{p}}=0.5 \mathrm{~km} \mathrm{~s}^{-1}, \\
\Delta T=0.2 \\
\psi=180^{\circ} \\
K=24 \\
\Sigma=0.0040 \text { continuum units. }
\end{array}\right.
$$

We note that the magnitude amplitude corresponding to this solution is about 0.02 , which is compatible with the observed one. The fit for these parameters is shown in Fig. 4. This solution leads to the same line-profile variations as the solution without the inclusion of temperature effects and is not able to reproduce the large observed equivalent width variation. The theoretical profiles were

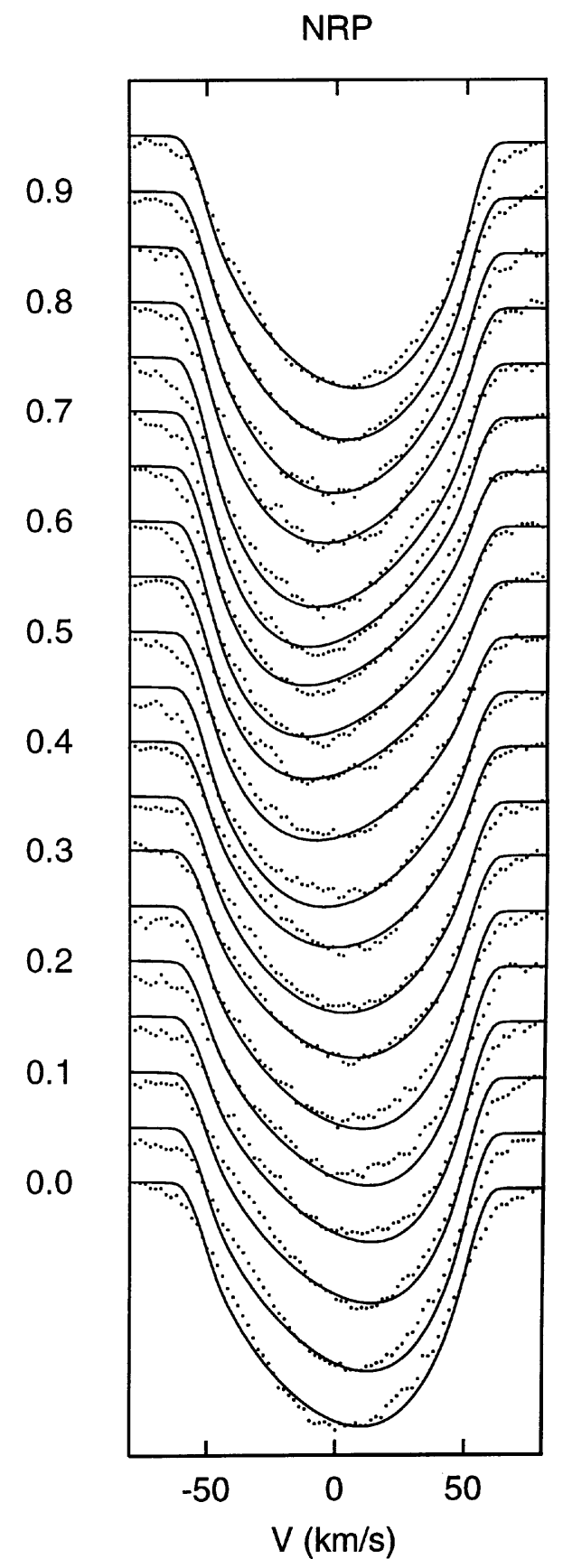

Fig. 4. Observed line profiles of the Si II $4128 \AA$ line (dots) averaged over phase bins of 0.05 and theoretical line profiles (full lines) for the NRP model with $(\ell, m)=(2,0)$.

adjusted to have the same equivalent width as the observed profiles. We note that this solution is not very different than the others in Table 4 and leads to the same value of $\Sigma$. Thus we have shown that the pulsation hypothesis does not agree well with the data.

\section{Rotational modulation model}

\subsection{A Bp star}

We searched the literature for chemical inhomogeneities in this star. Hiltner et al. (1969) indeed classified HD 131120 
as a Bp star and reported spectral peculiarities similar to those found in 3 Sco and HD 144334. These latter stars appear to be He-weak Si stars (see Garrison 1967; Norris 1971). The average equivalent width of the He $4121 \AA$ line measured in our data is about $68.9 \mathrm{~m} \AA$. By comparing this value with the ones for normal B 2.5 stars in Didelon (1982), we can confirm that HD 131120 is a He-weak star. We note that, in SIMBAD, we find a MK spectral type of B 7, which is misleading as it relies on He line strengths. The average equivalent width of the $\mathrm{Si} 4128 \AA$ line is about $95.7 \mathrm{~m} \AA$ which is quite normal for a B 2.5 star.

The chemically peculiar Bp stars show monoperiodic variations. Where light and line-profile variations are present, the same frequency is found in both data sets. The observed periods range from 1 to 20 days in the majority of cases. The periods show an inverse correlation with the projected rotational velocity. All these characteristics indicate that the variations of $\mathrm{Bp}$ stars are due to rotational modulation. Moreover, up to now, the variability of He-weak stars is explained by the rotation of the star in the presence of a non homogeneous distribution of helium on the stellar surface.

\subsection{Model with two spots}

We compared the line-profile observations of HD 131120 with a rotational modulation model by using a code kindly put at our disposal by Dr. L. Balona. This code calculates line-profile variations for a spotted star.

The following parameters are needed to construct lineprofile variations caused by a circular spot: the equatorial and polar radii $R_{\mathrm{e}}$ and $R_{\mathrm{p}}$, the equatorial and polar fluxes $F_{\mathrm{e}}$ and $F_{\mathrm{p}}$, the projected rotational velocity $v_{\Omega}$, the angle of inclination $i$, the linear limb-darkening coefficient $u$, the intrinsic line-profile width in the photosphere $\sigma_{i}$, the longitude (relative to some arbitrary epoch) $\lambda$, the latitude $\beta$, the spot radius in degrees $\gamma$, the flux from the spot relative to the photosphere $F$, the intrinsic line-profile width in the spot $\sigma_{\mathrm{s}}$. We take the equatorial and polar radii $R_{\mathrm{e}}=R_{\mathrm{p}}=3.6 R_{\odot}$, the equatorial and polar fluxes $F_{\mathrm{e}}=F_{\mathrm{p}}=1$ and the linear limb-darkening coefficient $u=0.36$. The other parameters are free parameters.

As HD 131120 appears to be a He-weak star, it was important to test the rotational modulation model also on the He $4121 \AA$ line. First we computed the first three moments of this line. The frequency search leads to $f_{1}=$ $0.6375 \mathrm{c} / \mathrm{d}$ and $f_{2}=2 f_{1}=1.275 \mathrm{c} / \mathrm{d}$ for $\left\langle v^{1}\right\rangle$ and $\left\langle v^{3}\right\rangle$. No frequency can be found for $\left\langle v^{2}\right\rangle$. The frequency $f_{1}$ and its first harmonic $f_{2}$ reduce the standard deviation by $53 \%$ for $\left\langle v^{1}\right\rangle$ and by about $37 \%$ for $\left\langle v^{3}\right\rangle$. A phase diagram for the frequency $f_{1}=0.6375 \mathrm{c} / \mathrm{d}$ for $\left\langle v^{1}\right\rangle,\left\langle v^{2}\right\rangle$ and $\left\langle v^{3}\right\rangle$ is shown in Fig. 5. Figure 6 shows that the $E W$ also varies with the same frequency. The relative $E W$ variation of the HeI line is about $16 \%$.

We point out that the first moment of the Si II $4128 \AA$ line and of the He $4121 \AA$ line do not have the same form. This observation is clearly an argument against a NRP
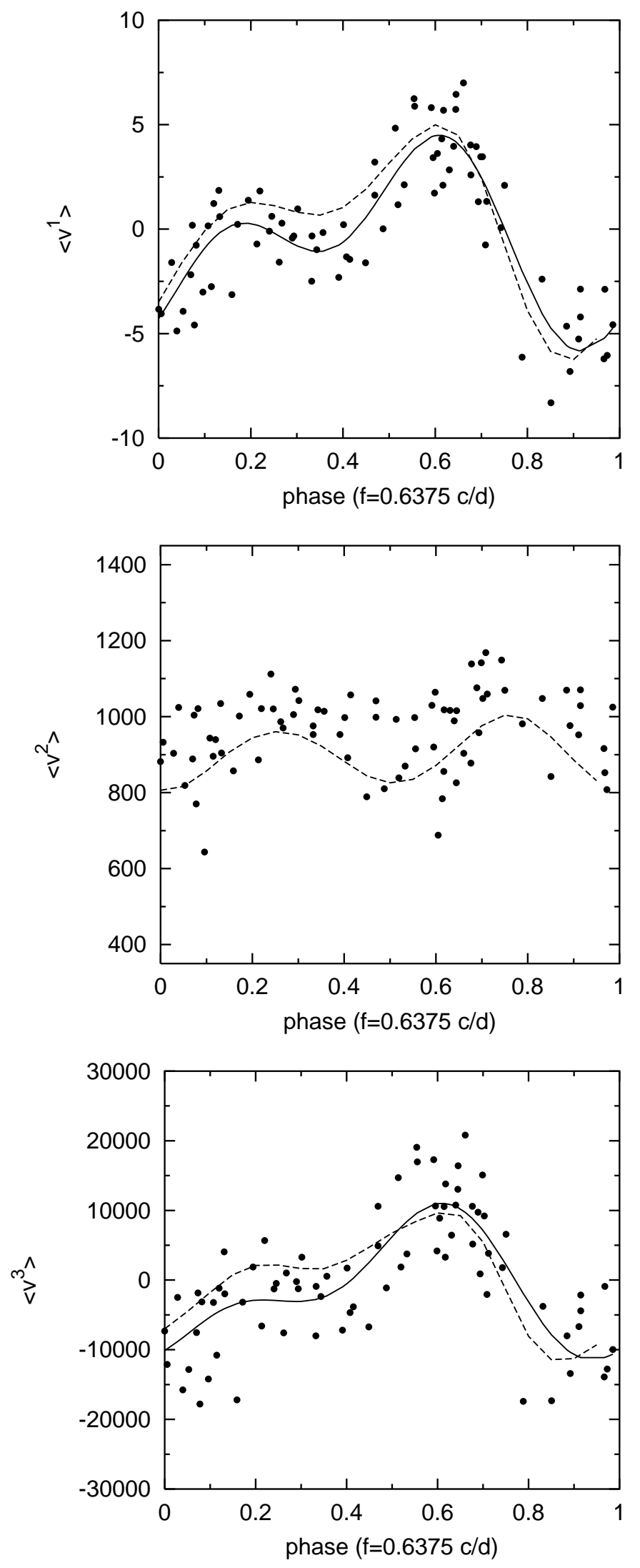

Fig. 5. Phase diagram of the first three moments of the He $4121 \AA$ line. We show the observed values (dots), the fit using $0.6375 \mathrm{c} / \mathrm{d}$ and its first harmonic (solid line), and the best spot model (dashed line). 


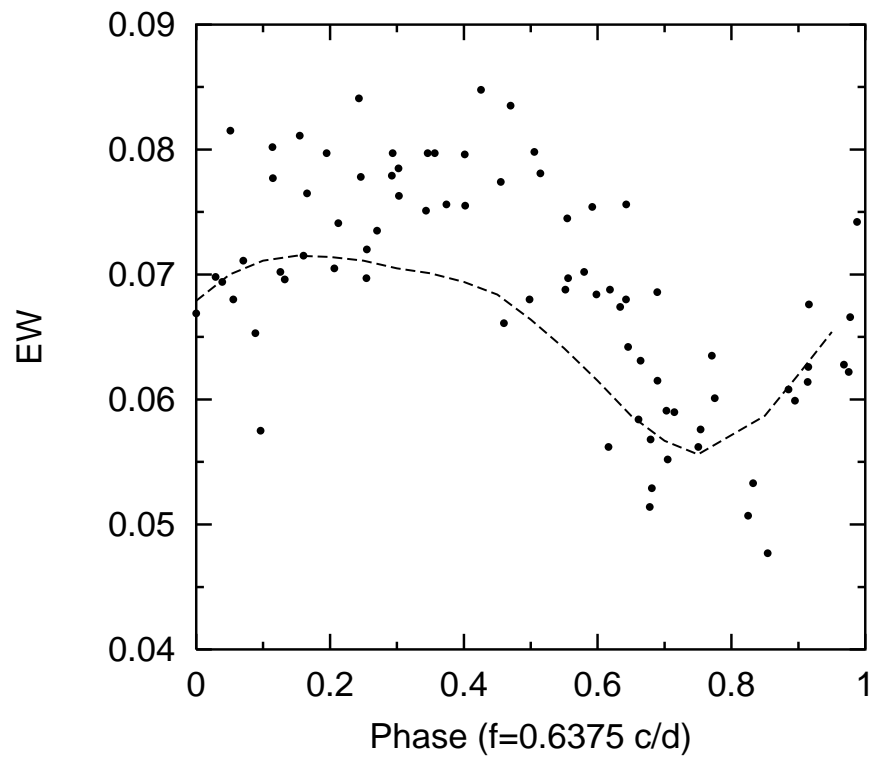

Fig. 6. Phase diagram of the equivalent width of the He $4121 \AA$ line, which is expressed in $\AA$. The comparison with the best spot model is represented in dashed line.

model. Morever, they are not in phase. This is not compatible with a NRP model.

Such variations can be reproduced by a spot model if the difference of longitude between a spot of Si and a spot of He is $180^{\circ}$. They can also appear if for one spot silicon is overabundant and helium is underabundant or silicon is underabundant and helium is overabundant.

A non-sinusoidal first moment with $f$ and $2 f$ can be obtained if we consider two spots of the same element, which have a longitude difference of $180^{\circ}$. Two spots give 13 free parameters, which leads to an enormous computational time. In order to reproduce the form of the first moment, we decided to test the two following cases:

$\left\{\begin{array}{l}\lambda_{1}=\lambda_{2}+180^{\circ}, \\ \beta_{1}=\beta_{2}, \\ \gamma_{1}=\gamma_{2}, \\ F_{1} \neq F_{2}, \\ \sigma_{\mathrm{s} 1}=\sigma_{\mathrm{s} 2},\end{array}\right.$

and

$\left\{\begin{array}{l}\lambda_{1}=\lambda_{2}+180^{\circ}, \\ \beta_{1}=\beta_{2}, \\ \gamma_{1} \neq \gamma_{2}, \\ F_{1}=F_{2}, \\ \sigma_{\mathrm{s} 1}=\sigma_{\mathrm{s} 2},\end{array}\right.$

where the indices 1 and 2 are respectively for the first spot and the second spot. We compared such a spot model to both the He line and the Si line. We again determine the parameters for which the theoretical profiles best fit the observations by minimizing the standard deviation $\Sigma$ in the intensity over all profiles as done for the fitting with BRUCE. Then the parameters were varied around the best set of parameters in order to refine the solution.
For the He line, the following parameters lead to a good fit, as we can see in Fig. 7:

$$
\left\{\begin{array}{l}
\lambda_{1}=90^{\circ}, \lambda_{2}=280^{\circ}, \\
\beta_{1}=50^{\circ}, \beta_{2}=80^{\circ} \\
\gamma_{1}=60^{\circ}, \gamma_{2}=6^{\circ} \\
F_{1}=0.35, F_{2}=0.4 \\
v_{\Omega}=54 \mathrm{~km} \mathrm{~s}^{-1} \\
i=30^{\circ} \\
\sigma_{i}=12 \mathrm{~km} \mathrm{~s}^{-1} \\
\sigma_{\mathrm{s}}=12 \mathrm{~km} \mathrm{~s}^{-1} \\
\Sigma=0.0022 \mathrm{continuum} \mathrm{units.}
\end{array}\right.
$$

In Fig. 5 the first three moments of the theoretical profiles shown in Fig. 7 are compared to the ones of the observed profiles of He. In Fig. 6 the variation of the theoretical $E W$ is compared to the observed $E W$ variation. It results that the behaviour of the observed moments is well reproduced by the theoretical moments.

For the Si line, we also obtain a good fitting with these parameters (see Fig. 7):

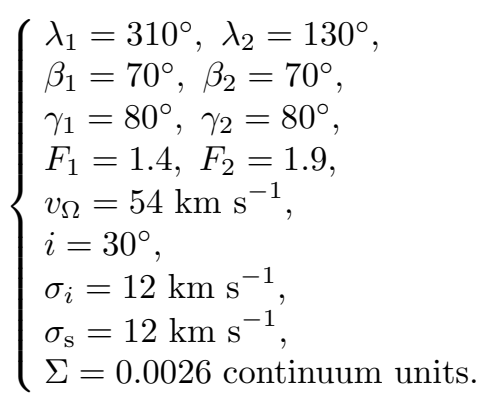

In Fig. 2 the first three moments of the theoretical profiles are compared to those of the observed profiles of $\mathrm{Si}$ and in Fig. 3 we show the comparison between the observed and theoretical $E W$ variations of the Si line. Again we find a good agreement between observed moments and theoretical moments. We note that the amplitude of the theoretical second moment is very small, which is compatible with the fact that the observed second moment is very noisy.

We point out that the models for the He line and for the Si line are compatible since they have the same value for the parameters $v_{\Omega}, i, \sigma_{i}$. We also point out that we end up with a model for which helium is underabundant in the spots while silicon is overabundant there. This naturally explains the very weak He line and the very strong Si line.

It would be interesting to compute the variation of the luminosity of HD 131120 in order to compare it to the observed photometric amplitudes. As we do not know if the star presents additional non homogeneous distributions of other elements on the stellar surface, we cannot compute this variation as long as we do not have a complete view of the chemical abundances.

\section{Conclusions}

Our study of the star HD 131120 was based on multicolour photometry and high-resolution spectroscopy spread over 
He 4121

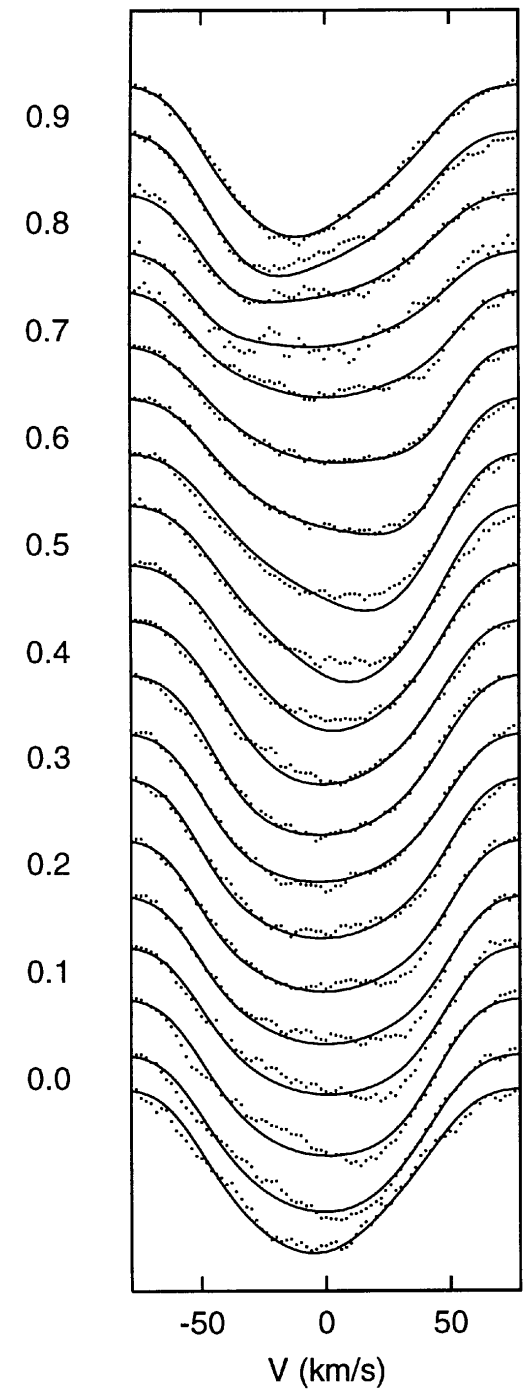

Si 4128

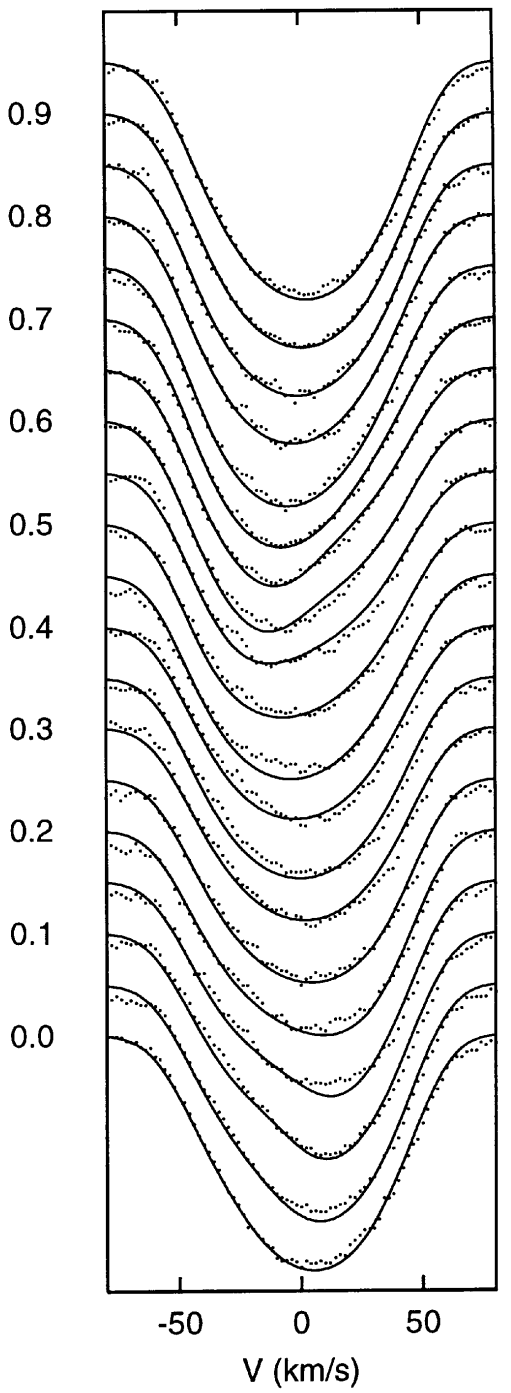

Fig. 7. Observed line profiles (dots) of the He $4121 \AA$ line (left) and of the Si II $4128 \AA$ line (right) averaged over phase bins of 0.05 and theoretical line profiles (full lines) for the spot model with two spots.

respectively one and two years. From the period analysis on all the data it is clear that this star is monoperiodic with a period of 1.569 days. We compared the variations in the data with a non-radial pulsation model. The mode identification by three methods does not allow to choose one particular pulsation mode. Moreover, the line-profile behaviour of the Si and He lines is very different as far as the temporal behaviour and phasing is concerned. Such a difference cannot be explained by a pulsational model.

Next we tried to interpret the monoperiodic variations by rotational modulation. We compared the line-profile variations with a model with two spots in order to reproduce the first moment of the observed spectral lines. This leads to very good results for both $\mathrm{He}$ and $\mathrm{Si}$ lines. Moreover, the best spot model is able to reproduce the behaviour of the first three moments of the lines, as well as the large equivalent width variations. Moreover, we showed that a spot model can well explain the different temporal behaviour of the moments of the two different lines. This work results in a model for a He-underabundant and Si-overabundant star, which is fully compatible with results reported in the literature. We conclude that rotational modulation is the best explanation of the variability of HD 131120 and remove the star from the list of SPBs. From the estimated $i, v_{\Omega}$ and frequency we derive a stellar radius of $3.4 R_{\odot}$, which is compatible with the value derived from photometry.

In the sample of selected SPBs, three other stars have moments that vary in a similar way to those of HD 131120. Two of these stars were not reported before as Bp stars. We plan to test the rotational modulation model for these stars as well in order to try to explain their variability in full detail. The outcome of this study will be given in a forthcoming paper. 
With this study we provide a very convenient way to discriminate between stellar pulsation and stellar spots. Indeed, the moment variations of the line profiles of different spectral lines have the same behaviour, as far as amplitudes and phases are concerned, when one is dealing with a pulsating star. For spotted stars, the moments of the line profiles clearly differ for different lines. Additionally, we have shown that the moment variations of the spectral lines of spotted stars allow one to derive very accurately the geometry and other physical characteristics of the surface spots.

Acknowledgements. We thank the referee, Dr. R. J. Dukes Jr., for his useful suggestions, which helped us to improve this paper. We used the SIMBAD astronomical database operated at the CDS in Strasbourg, France. This work was supported by the P. A. I. (Pôle d'Attraction Interuniversitaire).

\section{References}

Aerts, C., De Pauw, M., \& Waelkens, C. 1992, A\&A, 266, 294 Aerts, C. 1996, A\&A, 314, 115

Aerts, C., De Cat, P., Peeters, E., et al. 1999, A\&A, 343, 872 Aerts, C., De Cat, P., De Ridder, J., et al. 2000, in The Impact of Large-Scale Surveys on Pulsating Star Research, ed. L. Szabados, \& D. Kurtz., IAU Colloq. 176, ASP Conf. Ser., 203, 395
Borra, E. F., Landstreet, J. D., \& Thompson, I. 1983, ApJS, 53,151

Briquet, M., Aerts, C., \& De Cat, P. 2001, A\&A, 366, 121

De Cat, P., Aerts, C., De Ridder, J., et al. 2000, A\&A, 355, 1015

De Cat, P. 2001, Ph.D. Thesis, Katholieke Universiteit Leuven, Belgium

De Geus, E. J., de Zeeuw, P. T., \& Lub, J. 1989, A\&A, 216, 44

Didelon, P. 1982, A\&AS, 50, 199

Garrison, R. F. 1967, ApJ, 147, 1003

Gummersbach, C. A., Kaufer, A., Schäfer, D. R., Szeifert, T., \& Wolf, B. 1998, A\&A, 338, 881

Heynderickx, D., Waelkens, C., \& Smeyers, P. 1994, A\&AS, 105,447

Hiltner, W. A., Garrison, R. F., \& Schild, R. E. 1969, ApJ, 157,313

Norris, J. 1971, ApJS, 23, 213

Pamyatnykh, A. A. 1999, Acta Astronomica, 49, 119

Roberts, D. H., Lehár, J., \& Dreher, J. W. 1987, AJ, 93, 968

SAS/ User's Guide, 1990, Version 6, volumes 1-2, SAS Institute Inc., Cary, NC

Stamford, P. A., \& Watson, R. D. 1981, Ap\&SS, 77, 131

Stellingwerf, R. F. 1978, ApJ, 224, 953

Townsend, R. H. D. 1997, MNRAS, 284, 839

Waelkens, C., Aerts, C., Kestens, E., et al. 1998, A\&A, 330, 215

Watson, R. D. 1988, Ap\&SS, 140, 255 